\title{
One follicle, one egg, one embryo: a case-report of successful pregnancy obtained from a single oocyte collected
}

\author{
Romualdo Sciorio ${ }^{1,2}$, Elisavet Angelaki², Naser Al-Azemi², Abubakr Elmardi² \\ ${ }^{1}$ Edinburgh Assisted Conception Programme, EFREC, Royal Infirmary of Edinburgh, 51 Little France Crescent, \\ Old Dalkeith Road, Edinburgh, Scotland, EH164SA, UK \\ 2Reproductive Medicine \& IVF Unit, Royale Hayat Hospital, Kuwait City, Kuwait
}

\begin{abstract}
In this report, we present an unusual case of a couple who achieved a successful pregnancy by ICSI with a single oocyte collected. The cumulus-oocyte-complex (COC) was retrieved at 36.5 hours post trigger, and was found to be at metaphase II, when sperm injection was performed at around 39 hours post trigger. At 18 hours post injection, the single oocyte was fertilized, developed to four-cell embryo on day 2 , and 8 -cell grade on day 3 , when it was relocated in the uterine cavity. The pregnancy yielded a positive $\beta$-hCG result. The scan performed at seven weeks, revealed the presence of one amniotic sac with a fetal heartbeat. The ongoing pregnancy has been eventless at 24 weeks of gestation.
\end{abstract}

Keywords: in vitro fertilization (IVF), Cumulus-oocytecomplex (COC), ICSI time, Fertilization, Ongoing pregnancy

\section{Introduction}

Over eight million in vitro fertilization (IVF) children have been born since 1978, when Steptoe and Edwards (1978) announced the first IVF baby. Worldwide, approximately 2.5 million medically assisted reproduction (MAR) cycles are performed annually, resulting in over 500,000 deliveries. During the last four decades, IVF techniques have improved steadily, thus providing better pregnancy prospects for couples suffering from infertility. However, the in vitro development of human embryos is still suboptimal, and many good quality embryos fail to implant and generate a viable pregnancy (Hu et al., 1998).

The introduction of intracytoplasmic sperm injection (ICSI) (Palermo et al., 1992) has revolutionized the treatment of male infertility, in particular, for patients with low sperm counts. The ejaculate of recent fathers contains approximately 73 million spermatozoa (Esteves et al., 2012). According to the latest World Health Organization (WHO) manual for the examination of human semen, a sperm count of over 15 million/ml is considered normal (Cooper et al., 2010), with lower thresholds (termed oligozoospermia) associated with an increased risk of infertility. In theory, a single spermatozoon would be needed to fertilize an oocyte and generate a successful pregnancy using the ICSI procedure. Thus, in most oligozoospermic patients, sperm are retrieved successfully from their ejaculated samples collected on the day of egg retrieval.

However, the number of oocytes collected is a major determinant of IVF success, with higher yields for better outcomes (Esteves et al., 2019a). Thus, controlled ovarian stimulation (COS) using exogenous gonadotropins to stimulate the ovary is used to promote multifollicular development, with the aim of harvesting multiple COC, which are collected surgically. A pharmacological dose of FSH is typically used to induce the growth of multiple ovarian follicles. As follicles grow and reach the requisite size, LH exposure is provided to simulate the mid-cycle LH surge, which induces the process of oocyte maturation and subsequent ovulation (Shoham et al., 1995). Oocyte retrieval is precisely timed following the provision of LH exposure to retrieve mature oocytes before ovulation. $\mathrm{LH}$ exposure initiates the resumption of meiosis and the oocyte maturation from the "metaphase I" (MI) stage to the mature "metaphase II" (MII) stage of development. During this maturation process, the first polar body is extruded, and the oocyte reaches the MII stage, which denotes its competence to be fertilized by a spermatozoon (Voronina \& Wessel, 2003). Following the LH-like exposure, the remainder of the follicle forms the corpus luteum, which produces sex steroids, particularly progesterone, to prepare the endometrium for embryo implantation (Palomba et al., 2015).

Given the expected low number of oocytes in patients with reduced ovarian reserve and advanced maternal age, several strategies have been applied to raise the number of follicles and oocytes collected, including the increased gonadotropin dose. The classic treatment remains the COS, using the long GnRH agonist protocol, associated with hCG trigger for oocyte maturation (Chen et al., 2015). Despite that, in some patients undergoing IVF, only a limited number of oocytes are retrieved after COS. In this case, the likelihood of having a fertilized oocyte and a further transferable embryo with a successful pregnancy is very low, especially in women with advanced maternal age.

\section{Case presentation}

A married couple was referred to the reproductive medicine department at Royale Hayat Hospital, Kuwait city, for ICSI treatment in 2019. They presented with a four years' history of male infertility and low ovarian reserve. The husband's age was 25 years and his semen analysis showed oligo-astheno-teratozoospermia. His wife was a 39 years-old lady with regular ovulatory cycles and FSH levels of $8.79 \mathrm{mIU} / \mathrm{mL}$, and BMI of 29. They underwent a full ICSI cycle, and we counseled the couple regarding the need for ICSI treatment given the quality of sperm in the ejaculate. The woman responded poorly to the COS. Briefly, 450 IU daily of human gonadotropins (Gonal-F, Merck, Rome, Italy) were administrated for twelve days (total gonadotrophin dose of $5.400 \mathrm{IU}$ ). One dominant follicle was seen on the right ovary, whereas no follicles were seen on the left ovary. Oocyte maturation was triggered by using a single injection of 15.000 IU IVF-C (Human Chorionic Gonadotropin, Ciplamed) subcutaneously. Transvaginal ultrasound-guided oocyte retrieval was carried out 36.5 hours after the trigger. Only one COC was retrieved during 
the oocyte pick up. On that day, the sperm concentration was 5.8 million/ml, the total motility was $25 \%$, and the strict morphology $1 \%$.

\section{Oocyte retrieval, ICSI, Embryo culture and Transfer \\ The cumulus-oocyte-complex ( $\mathrm{COC}$ ) was isolated} from follicular fluid and then rinsed in $1.0 \mathrm{ml}$ G-MOPS ${ }^{\mathrm{TM}}$ plus medium (Vitrolife, Göteborg, Sweden). Following the oocyte pick-up, the oocyte was transferred to a $1.0 \mathrm{ml}$ equilibrated G-IVF ${ }^{\mathrm{TM}}$ medium (Vitrolife) at $37^{\circ} \mathrm{C}$, and $6 \%$ $\mathrm{CO}_{2}, 5 \% \mathrm{O}_{2}$ and nitrogen balance in a $\mathrm{K}$-System incubator (K-System G210, CooperSurgical, Inc. USA) until the time of ICSI. We collected the sperm used for the ICSI procedure by masturbation, and processed it using the density gradient technique with the following steps: first, a gradient column was prepared by placing $0.6 \mathrm{ml} 90 \%$ gradient media (Vitrolife, Sweden) in a centrifuge tube and an additional $0.6 \mathrm{ml} 45 \%$ gradient media layered on top. Next, up to $1.2 \mathrm{ml}$ of the semen was layered on top of the $55 \%$ layer and centrifuged at $1.600 \mathrm{rpm}$ for 15 minutes. The supernatant and gradient medium just above the sperm pellet were removed and discarded. The sperm pellet was washed with $6 \mathrm{ml} \mathrm{G-MOPS}{ }^{\mathrm{TM}}$ plus medium (Vitrolife, Sweden) and centrifuged at $1.400 \mathrm{rpm}$ for 10 minutes. The supernatant was discarded and the pellet was resuspended in $0.1 \mathrm{ml}$ G-MOPS ${ }^{\mathrm{TM}}$ plus medium. Approximately 39 hours after trigger, the oocyte was treated with hyaluronidase $(80 \mathrm{mIU} / \mathrm{ml})$ for $45-60$ seconds in order to remove the surrounding cumulus cells. At that time, the oocyte was found to be at the MII stage, with clear extrusion of the first polar body.

A single spermatozoon with normal morphology and progressive motility was selected under an inverted microscope (Nikon Eclipse Ti-S, Japan), and microinjected with the use of electrohydraulic injectors (Narishige, Japan). The oocyte was kept still by using a holding micropipette at the 9 o'clock position, and the polar body was oriented at the 12 o'clock position. The injecting pipette was then gently advanced through the zona pellucida and oolemma, until the pipette was beyond the center of the oocyte; then, the sperm was gently deposited into the oocyte's cytoplasm. The oocyte was examined for the presence of two pronuclei, and successful fertilization was confirmed at approximately 18 hours after insemination.

On day 2 , the embryo was a four-cell grade one, reaching eight cells grade one on day 3 , when it was transferred to the uterine cavity (Figure 1). The embryo culture was completed adopting a sequential preequilibrated medium (Vitrolife, G-series) as follows. Firstly, the fertilized oocyte was placed into a 20-microliter drop of $\mathrm{G}-1^{\mathrm{TM}}$ media, covered by light paraffin oil, sterile filtered (OVOIL ${ }^{\text {TM}}$ - Culture Oil, Vitrolife, Sweden). On the morning of day 3 , the embryo was transferred from the $\mathrm{G}-1^{\mathrm{TM}}$ micro droplet to a 20-microliter droplet of $\mathrm{G}-2^{\mathrm{TM}}$ medium, and kept in culture until the afternoon, when the embryo transfer was performed. We hereby report a compelling case in which an ongoing pregnancy with fetal heartbeat was obtained from the only oocyte collected after COS. The embryo generated by ICSI was transferred on day 3 (Figure 1). The embryo replacement was completed under transabdominal ultrasound guidance using a soft transfer catheter (Wallace ${ }^{\circledR}$ Classic, Cooper Surgical, USA). We started the luteal phase support with $90 \mathrm{mg}$ progesterone vaginal gel twice a day (Crinone $8 \%$, Merck) on the evening of egg collection day and continued till the $9^{\text {th }}$ gestational week, adding Progylutomn tablet $2 \mathrm{mg}$, Clexane $0.4 \mathrm{ml}$ and a tablet of baby Aspirin $75 \mathrm{mg}$ daily. At the embryo transfer, the patient started taking two injection weekly of Biosterone-Depot $250 \mathrm{mg}$ and continued until nine weeks of gestation.

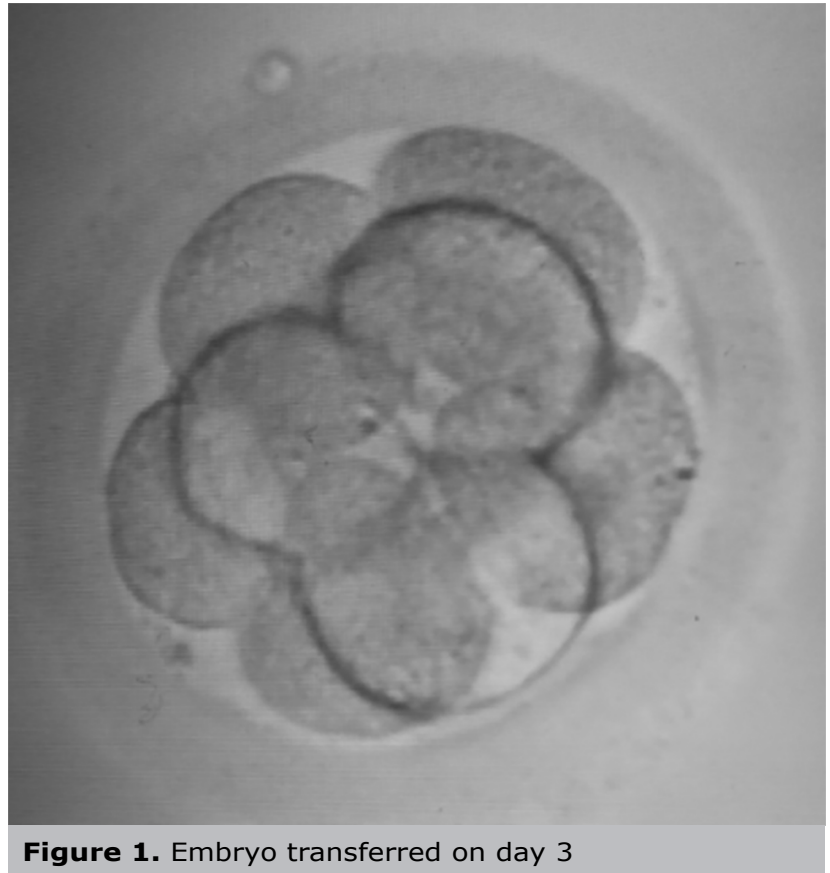

\section{Pregnancy outcome}

We implanted the embryo and seven weeks later, we performed the first scan, which revealed the presence of one amniotic sac with fetal heartbeat (Figure 2: ultrasound scan performed at 10 weeks of gestation). Currently the gestation is at 24 weeks, and so far it has been eventless and without any complications.

\section{DISCUSSION}

In the last few decades, the number of couples facing infertility has increased steadily, many of whom will ultimately need MAR treatments (Esteves et al., 2018). Global data reports over 8 million IVF babies born in the last 42 years, and in the UK, IVF babies account for about 3\% of all babies born in 2016 (Adamson et al., 2011; Human Fertilization \& Embryology Authority 2018; De Geyter et al., 2014). During the last 40 years, IVF techniques have improved steadily, thus providing better pregnancy prospects for couples suffering from infertility. However, despite the remarkable advances in reproductive technology, the development of human embryos in vitro is still suboptimal, and many good quality embryos fail to implant and generate a viable pregnancy (Hu et al., 1998).

IVF outcome is dependent on multiple factors, including oocyte and sperm quality, maternal age, infertility cause, lifestyle factors, as well as laboratory conditions for manipulation and embryo culture (Sciorio \& Smith, 2019; Gardner, 2016). Appropriate oocyte cytoplasmic and nuclear maturation are paramount to ensure an optimal embryonic developmental competence. While nuclear maturation is usually obtained by the time of oocyte retrieval, the cytoplasmic maturation cannot be readily assessed and might be incomplete (Voronina \& Wessel, 2003). Certainly, the oocyte must be at the MII stage, which denotes its competence to be fertilized by a spermatozoon. Therefore, several authors have reported that the waiting time of 2 or 4 hours between oocyte retrieval and insemination improves fertilization rate, embryo quality and pregnancy outcomes (Rienzi et al., 1998; Isiklar et al., 2004; Dozortsev et al., 2004). 


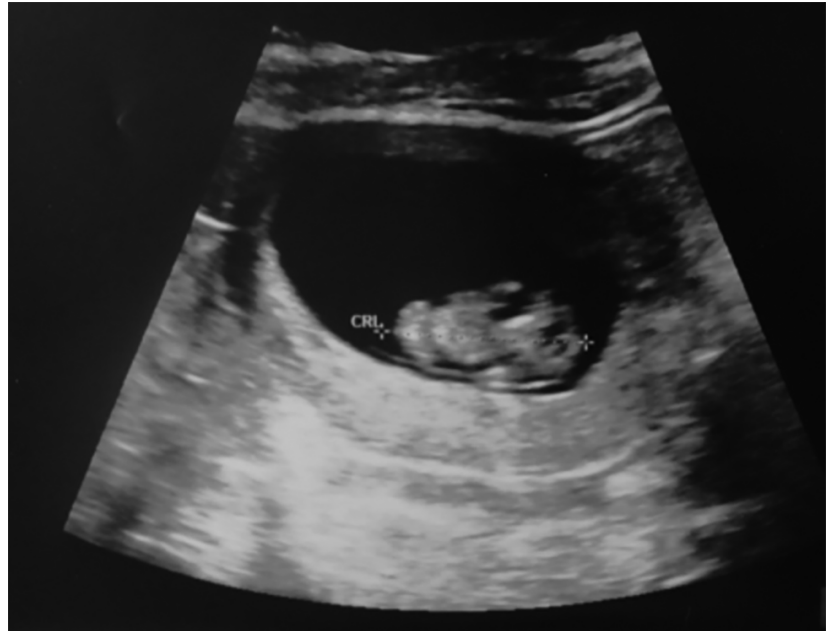

Figure 2. Picture shows the ultrasound scan performed at 10 weeks of gestation

On the other hand, excessive in vitro culture of human oocytes can affect their ultrastructural components, and have been linked to chromosomal and spindle abnormalities, mitochondrial damage and compromised pregnancy outcomes (Bianchi et al., 2015). However, a crucial aspect to notice is the oocyte number, which has a direct impact on the MAR treatment outcome. This aspect was investigated in a study published by Esteves et al. (2019). The authors evaluated the minimum oocyte number needed to achieve at least one euploid blastocyst for transfer. The model considered the female age, sperm source used for ICSI, and the number of mature (MII) oocytes as predictors. The final predictive model was developed using logistic regression analysis. The results found that the woman's age the most critical predictor for the likelihood of achieving one euploid blastocyst. The estimated predicted probabilities of a mature oocyte turn into a euploid blastocyst decreased progressively with female age and was negatively affected by use of testicular sperm across age. Therefore, in this case report, we were somehow surprised to see that the only COC collected from a 39 year old lady, was found to be at MII at ICSI time, fertilized and developed into a good quality embryo, which after transfer, resulted in a viable pregnancy.

Generally, the probability of having a good quality embryo to transfer after the retrieval of a single COC is low, even lower in women with advanced maternal age (AMA; > 35 years). Currently, no remedies are available to counteract the aging-related fertility decay; however, different therapeutic approaches, such as using high doses of gonadotropins to stimulate the ovary, like the one applied for the case reported here, might be offered. A recent paper published by Ubaldi et al., (2019) summarizes the current approaches for AMA patients undergoing MAR treatments. This case's unique characteristic highlights the importance of MAR to overcome the most adverse situations. Health professionals providing care to infertility patients should be aware that it is possible to provide couples, like the one described here, with a real hope of biological parenthood. Equally important is to share such cases within the MAR community, which is often skeptical about the likelihood of one collected oocyte to generate a viable embryo and pregnancy with fetal heartbeat, which currently have been eventless at 24 weeks of gestation.
Finally, it is important to mention, in a case like this one, it is important that the couple have appropriate counseling. With only one follicle available and one oocyte retrieved, it is crucial to inform the couple about the probability that the oocyte may not be fertilized, leaving no embryo for transfer. Discussing these data will help set reasonable expectations and enable quick movement to a future treatment.

\section{CONCLUSION}

We report here a compelling case in which a 39 yearold woman after COS, was able to produce one follicle. Approximately 36.5 hours after trigger, one oocyte was collected and then fertilized by ICSI. The fertilized embryo on day 3 was found to be at the eight-cell stage; it was relocated to the uterine cavity, and resulted in a successful pregnancy. Our case-report stimulates the discussion on the above aspects, and emphasize that, although live birth would have been the ultimate endpoint for such report, we do believe the ongoing pregnancy at 24 weeks to be relevant for stimulating the discussion of unusual aspects of this report.

\section{CONFLICT OF INTEREST}

The authors reported no conflict of interest.

\section{Corresponding author:}

Romualdo Sciorio

Edinburgh Assisted Conception Program, EFREC,

Royal Infirmary of Edinburgh

Edinburgh, Scotland, UK.

Reproductive Medicine \& IVF Unit

Royale Hayat Hospital

Kuwait City, Kuwait

Email: sciorioromualdo@hotmail.com

\section{REFERENCES}

Adamson GD, Mouzon J, Chambers GM, Zegers-Hochschild F, Mansour R, Ishihara O, Banker M, Dyer S. International Committee for Monitoring Assisted Reproductive Technology: world report on assisted reproductive technology, 2011. Fertil Steril. 2018;110:1067-80. PMID: 30396551 DOI: 10.1016/j.fertnstert.2018.06.039

Bianchi S, Macchiarelli G, Micara G, Linari A, Boninsegna C, Aragona C, Rossi G, Cecconi S, Nottola SA. Ultrastructural markers of quality are impaired in human metaphase II aged oocytes: a comparison between reproductive and in vitro aging. J Assist Reprod Genet. 2015;32:1343-58. PMID: 26276431 DOI: 10.1007/ s10815-015-0552-9

Chen $\mathrm{H}$, Wang $\mathrm{Y}$, Lyu Q, Ai A, Fu $\mathrm{Y}$, Tian $\mathrm{H}$, Cai R, Hong Q, Chen Q, Shoham Z, Kuang Y. Comparison of livebirth defects after luteal-phase ovarian stimulation vs. conventional ovarian stimulation for in vitro fertilization and vitrified embryo transfer cycles. Fertil Steril. 2015;103:1194-201.e2. PMID: 25813280 DOI: 10.1016/j. fertnstert.2015.02.020

Cooper TG, Noonan E, Von Eckardstein S, Auger J, Baker HW, Behre HM, Haugen TB, Kruger T, Wang C, Mbizvo MT, Vogelsong KM. World Health Organization reference values for human semen characteristics. Hum Reprod Update. 2010;16:231-45. PMID: 19934213 DOI: 10.1093/ humupd/dmp048 
De Geyter C, Calhaz-Jorge C, Kupka MS, Wyns C, Mocanu E, Motrenko T, Scaravelli G, Smeenk J, Vidakovic S, Goossens V; The European IVF-monitoring Consortium (EIM) for the European Society of Human Reproduction and Embryology (ESHRE). ART in Europe, 2014: results generated from European registries by ESHRE: The European IVFmonitoring Consortium (EIM) for the European Society of Human Reproduction and Embryology (ESHRE). Hum Reprod. 2018;33:1586-601. PMID: 30032255 DOI: $10.1093 /$ humrep/dey242

Dozortsev D, Nagy P, Abdelmassih S, Oliveira F, Brasil A, Abdelmassih V, Diamond M, Abdelmassih R. The optimal time for intracytoplasmic sperm injection in the human is from 37 to 41 hours after administration of human chorionic gonadotropin. Fertil Steril. 2004;82:1492-6. PMID: 15589848 DOI: $10.1016 /$ j.fertnstert.2004.09.002

Esteves SC, Zini A, Aziz N, Alvarez JG, Sabanegh Junior ES, Agarwal A. Critical appraisal of World Health Organization's new reference values for human semen characteristics and effect on diagnosis and treatment of subfertile men. Urology. 2012;79:1622. PMID: 22070891 DOI: 10.1016/j.urology.2011.08.003

Esteves SC, Roque M, Bedoschi G, Haahr T, Humaidan P. Intracytoplasmic sperm injection for male infertility and consequences for offspring. Nat Rev Urol. 2018;15:53562. PMID: 29967387 DOI: $10.1038 / s 41585-018-0051-8$

Esteves SC, Alviggi C, Humaidan P, Fischer R, Andersen CY, Conforti A, Bühler K, Sunkara SK, Polyzos NP, Galliano D, Grynberg M, Yarali H, Özbek IY, Roque M, Vuong LN, Banker M, Rienzi L, Vaiarelli A, Cimadomo D, Ubaldi FM. The POSEIDON criteria and its measure of success through the eyes of clinicians and embryologists. Front Endocrinol. 2019a; 10:814. PMID: 31824427 DOI: 10.3389/fendo.2019.00814

Esteves SC, Carvalho JF, Bento FC, Santos J. A novel predictive model to estimate the number of mature oocytes required for obtaining at least one euploid blastocyst for transfer in couples undergoing in vitrofertilization/intracytoplasmic sperm injection: the ART calculator. Front Endocrinol. 2019b;10:99. PMID: 30873117 DOI: 10.3389/fendo.2019.00099

Gardner DK. The impact of physiological oxygen during culture, and vitrification for cryopreservation, on the outcome of extended culture in human IVF. Reprod Biomed Online. 2016;32:137-41. PMID: 26687905 DOI: 10.1016/j.rbmo.2015.11.008

Hu Y, Maxson WS, Hoffman DI, Ory SJ, Eager S, Dupre J, Lu C. Maximizing pregnancy rates and limiting higher-order multiple conceptions by determining the optimal number of embryos to transfer based on quality. Fertil Steril. 1998;69:650-7. PMID: 9548153 DOI: 10.1016/S0015-0282(98)00024-7
Human Fertilisation \& Embryology Authority (HFEA) [Internet]. Fertility treatment 2014-2016: trends and figures. London: HFEA; 2018. Available at: https:// www.hfea.gov.uk/media/3188/hfea-fertility-trendsand-figures-2014-2016.pdf.

Isiklar A, Mercan R, Balaban B, Alatas C, Aksoy S, Urman B. Impact of oocyte pre-incubation time on fertilization, embryo quality and pregnancy rate after intracytoplasmic sperm injection. Reprod Biomed Online. 2004;8:682-6. PMID: 15169586 DOI: 10.1016/ S1472-6483(10)61649-5

Palermo G, Joris H, Devroey $P$, Van Steirteghem AC. Pregnancies after intracytoplasmic injection of single spermatozoon into an oocyte. Lancet. 1992;340:17-8. PMID: 1351601 DOI: 10.1016/0140-6736(92)92425-F

Palomba S, Santagni S, La Sala GB. Progesterone administration for luteal phase deficiency in human reproduction: an old or new issue? J Ovarian Res. 2015;8:77. PMID: 26585269 DOI: 10.1186/s13048-0150205-8

Rienzi L, Ubaldi F, Anniballo R, Cerulo G, Greco E. Preincubation of human oocytes may improve fertilization and embryo quality after intracytoplasmic sperm injection. Hum Reprod. 1998;13:1014-9. PMID: 9619563 DOI: 10.1093/humrep/13.4.1014

Sciorio R, Smith GD. Embryo culture at a reduced oxygen concentration of 5\%: a mini review. Zygote. 2019;27:355- 361. PMID: 31544720 DOI: 10.1017/ S0967199419000522

Shoham Z, Schacter M, Loumaye E, Weissman A, MacNamee $M$, Insler $V$. The luteinizing hormone surge-the final stage in ovulation induction: modern aspects of ovulation triggering. Fertil Steril. 1995;64:237-51. PMID: 7615097 DOI: $10.1016 /$ s0015-0282(16)57717-6

Steptoe PC, Edwards RG. Birth after the reimplantation of a human embryo. Lancet. 1978;2:366. PMID: 79723 DOI: $10.1016 / \mathrm{s} 0140-6736(78) 92957-4$

Ubaldi FM, Cimadomo D, Vaiarelli A, Fabozzi G, Venturella R, Maggiulli R, Mazzilli R, Ferrero S, Palagiano A, Rienzi L. Advanced maternal age in IVF: still a challenge? The present and the future of its treatment. Front Endocrinol. 2019;10:94. PMID: 30842755 DOI: 10.3389/ fendo.2019.00094

Voronina $E$, Wessel GM. The regulation of oocyte maturation. Curr Top Dev Biol. 2003;58:53-110. PMID: 14711013 DOI: $10.1016 /$ s0070-2153(03)58003-6 